This item was submitted to Loughborough's Research Repository by the author.

Items in Figshare are protected by copyright, with all rights reserved, unless otherwise indicated.

\title{
Rate-delay analysis of radio access network slices
}

PLEASE CITE THE PUBLISHED VERSION

https://doi.org/10.1109/GLOCOM.2018.8647758

PUBLISHER

(c) IEEE

VERSION

AM (Accepted Manuscript)

LICENCE

CC BY-NC-ND 4.0

REPOSITORY RECORD

Zhou, Guorong, Liqiang Zhao, Qiong Shi, Gan Zheng, and Kwang-Cheng Chen. 2019. "Rate-delay Analysis of Radio Access Network Slices". figshare. https://hdl.handle.net/2134/37584. 


\title{
Rate-Delay Analysis of Radio Access Network Slices
}

\author{
Guorong Zhou ${ }^{1}$, Liqiang Zhao ${ }^{1}$, Qiong Shi $^{1}$, Gan Zheng ${ }^{2}$, Kwang-Cheng Chen ${ }^{3}$ \\ 1. The State Key Laboratory of Integrated Services Networks, Xidian University, Xi'an, China \\ 2. University of Loughborough, Loughborough, UK \\ 3. Department of Electrical Engineering, University of South Florida, Tampa, USA \\ E-mail: \{guor_zhou@163.com,lqzhao@mail.xidian.edu.cn,q.shi@outlook.com,g.zheng@lboro.ac.uk,kwangcheng@usf.edu\}
}

\begin{abstract}
Based on wireless network virtualization, radio access network (RAN) slicing is developed to provide services for the different users' requirements. Moreover, the users' sum data rate and delay are two significant metrics to guarantee quality of services. In this paper, we first establish an optimization problem to maximize the downlink sum rate while guaranteeing users' delay for RAN slices, where the base stations and user equipments are randomly distributed. Then we analyze the performance tradeoff between the sum rate maximization and delay tolerance. With the aid of Lyapunov optimization, the upper bounds of the achievable rate and delay are derived, through which the existence of tradeoff in performance is obvious and verified by numerical results.
\end{abstract}

Index Terms-RAN slice; Sum rate; Delay; Lyapunov optimization

\section{INTRODUCTION}

To improve the flexibility of services for $5 \mathrm{G}$ systems, wireless network virtualization (WNV) has emerged to abstract the physical network resources into virtual resources to realize sharing [1], [2]. Further, with WNV, end-to-end network slicing from the device to the core network could be created in order to satisfy users' customized services [3]. Radio access network (RAN) slicing [4], as an important part of network slicing, specifically allows the virtual radio resources and virtual base stations (BSs) in RAN to be dynamically allocated to various services.

A lot of work has focused on the resource management and the analysis of its utility for RAN slices up to now. Specifically, the authors in [5] analyze the RAN slicing in a multi-cell network and its relationship to the radio resource management functionalities that can be used for splitting the radio resources among the RAN slices. And the authors in [6] consider a dynamic resource allocation criterion amongst tenants in RAN slices to optimize utility gains and capacity savings. However, there are still many other performance indicators for RAN slices remained to be investigated.

In particular, the ever-growing traffic demands and personalized user requests make the users' sum data rate one of the major quality of service (QoS) goals for RAN slices. To ensure the fairness of the resources allocation in virtual networks, for service providers, [7] maximizes network throughput and reduces service time. A dynamic network slicing scheme for multitenant heterogeneous cloud radio access networks is proposed in [8] to achieve a higher network throughput. Furthermore, delay is viewed as a critical performance indicator related to the system reliability, which also reflects users' QoS provided by RAN slices. A QoS model for a wireless virtualization network is developed in [9] to obtain the upper bound about network delay. [10] proposes the network slicing in fog radio access networks and formulates an average delay optimization problem for one slice with user data rate guarantee for the other slice. From another perspective, to achieve higher network efficiency, the joint analysis or tradeoff strike of performance indicators is very necessary in communication network. For instance, [11] and [12] study the performance tradeoff of the energy efficiency versus delay in WNV.

Despite existing research on RAN slices and the performance analysis in WNV, a holistic study on sum rate and delay tradeoff of RAN slices to better guarantee QoS is not available in the literature, which is the focus of this paper. Different from existing virtualization related works, our new RAN slice model is extended from single cell to multi-cell with randomly distributed BSs and user equipments (UEs) and the interference between cells is taken into account. Firstly, the optimization of the dynamic density of virtual BSs in RAN slices is investigated to maximize the sum rate while supporting users' delay tolerance. Secondly, since this problem is challenging due to the absence of a closed-form solution for the users' sum rate, we solve it by using the Lyapunov optimization, which reduces the implementation complexity effectively without loss of generality, because any prior knowledge of traffic rate is not required. Finally, the upper bounds of the delay and sum rate about user density, virtual BS density and a control factor are analytically derived to strike a tradeoff, and the impact of relevant parameters on the rate and delay performance is evaluated by numerical results.

\section{SySTEM ModeL}

\section{A. RAN Slices}

We consider the RAN slices, as shown in Fig. 1. In this network, there is a mobile network operator (MNO). The buffer resources in BSs and radio spectrum resources in Air Interfaces owned by the MNO can be abstracted into multiple 


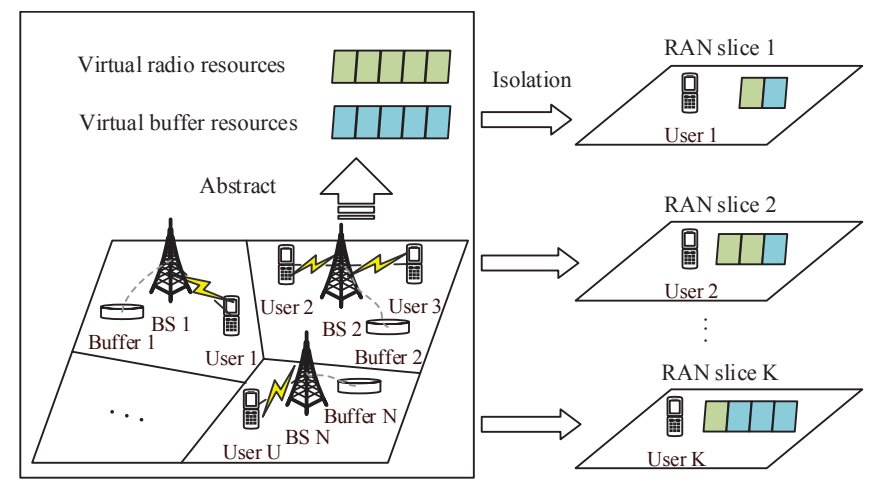

Figure 1. RAN slices.

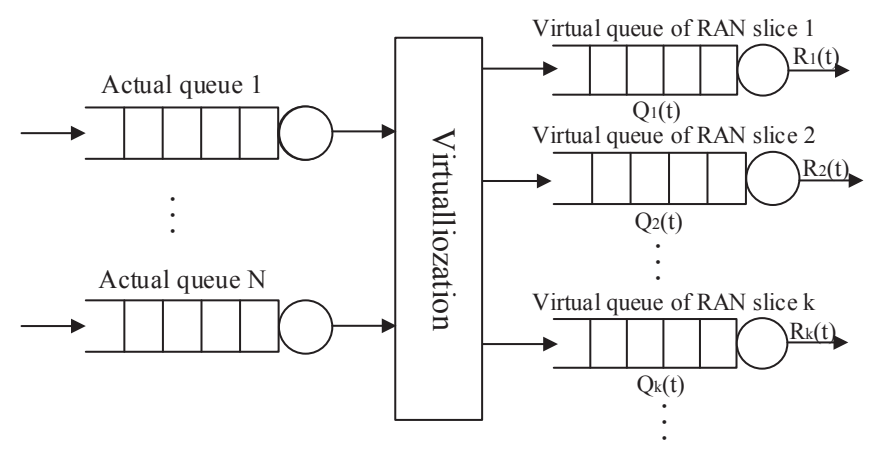

Figure 2. The actual queueing process and virtual queue representations in RAN slices.

virtual resource units in the virtual resource pool. Besides, a physical BS will be virtualized into one or several virtual BSs, while MNO determines the amount of virtual buffer and radio resource units allocated to each virtual BS based on diverse location information and QoS requirements of users, further to form customized RAN slices with isolation guarantee to provide users' access services. Once the user's rate and delay requests, or channel conditions in the current networks change, the virtual buffer and radio resource allocation of RAN slices will be changed.

Without loss of generality, we assume a downlink wireless network scenario in which $N$ physical BSs and multiple UEs are randomly distributed in the Euclidean plane with an area of $S_{\text {area }}$. Therefore, we use the stochastic geometry here to model the BSs and UEs deployment. Through virtualization, we obtain specific RAN slices based on users' location information and their customized QoS requirements. For simplicity, we suppose that each RAN slice is customized for one user. The system is operated time slotted and the slot is normalized to an integral unit described as a time interval $[t, t+1), t \in\{0,1,2, \ldots\}$.

As the virtual BSs and UEs distributions in RAN slices are time-varying due to the users' mobility and the users' customized requirements, the locations of virtual BSs and UEs are modeled by the dynamic homogeneous Possion point process (PPP) $\Phi_{V B}=\left\{b_{i}, i=0,1,2, \ldots\right\}$ with density $\lambda_{V B}(t)$ and $\Phi_{U}=\left\{u_{i}, i=0,1,2, \ldots\right\}$ with density $\lambda_{U}(t)$, respectively, where $t \in\{0,1,2, \ldots\}$. For simplification, it is assumed the virtual BSs and UEs deployment in our model is constant within a slot, and only changes from slot to slot. In addition, let vectors $A(t)=\left\{A_{1}(t), A_{2}(t), \ldots, A_{u}(t), \ldots\right\}$ and $Q(t)=\left\{Q_{1}(t), Q_{2}(t), \ldots, Q_{u}(t), \ldots\right\}$ denote the processes of random data arrivals and the current data queues in multiple RAN slices in slot $t$, respectively, where $A(t)$ is independent and identically distributed (i.i.d.) over time with the arrival rate $\gamma$.

\section{B. Rate Analysis of RAN slices}

The maximum transmission power of a physical BS is denoted by $P_{B}$, and the total power of the network is:

$$
P_{\text {sum }}=N \cdot P_{B}
$$

$P_{C}$ is used to indicate the circuit power consumption of a physical BS when there is no virtualization. Since virtualization consumes extra circuit power, the circuit power consumption of the virtual networks can be defined as:

$$
P_{V C}(\mathrm{t})=P_{C} \lambda_{V B}(t) S_{a r e a} .
$$

When the density of virtual BSs increases, the circuit power consumption by virtualization will also increase. In addition, the maximum transmit power of each virtual BS is as follows:

$$
P_{V B}(t)=\frac{N P_{B}-P_{C} \lambda_{V B}(t) S_{a r e a}}{\lambda_{V B}(t) S_{\text {area }}} .
$$

Then, we can define the time-average expectation of $P_{V B}(t)$ as follows:

$$
\bar{P}_{V B}\left(\lambda_{V B}(t)\right)=\lim _{t \rightarrow \infty} \frac{1}{t} \sum_{\tau=0}^{t-1} E\left\{P_{V B}(\tau)\right\} .
$$

Without loss of generality, each UE is associated with the closest virtual BS and $r_{u, \min }$ represents the distance between them. The power loss propagation is modeled by $h r^{-\alpha}$, where the small-scale channel gain $h$ follows an exponential distribution with unit mean. $\alpha>2$ and $r$ denote the path loss exponent and the propagation distance respectively. For a typical user $u$, the signal to interference plus noise ratio (SINR) can be expressed as:

$$
\begin{aligned}
& S I N R_{u}(t)=\frac{P_{V B}(t) h_{u, V B_{i}} r_{u, \text { min }}^{-\alpha}}{I_{u}(t)+\sigma^{2}} \\
& =\frac{\left(N P_{B}-P_{C} \lambda_{V B}(t) S_{a r e a}\right) h_{u, V B_{i}} r_{u, m i n}^{-\alpha}}{\lambda_{V B}(t) S_{\text {area }}\left(I_{u}(t)+\sigma^{2}\right)}, \forall u,
\end{aligned}
$$

where $h_{u, V B_{i}}$ is the channel gain between user $u$ and its nearest virtual BS $V B_{i}$ and $\sigma^{2}$ is the noise power. $I_{u}(t)$ is the interference from other virtual BSs, denoted as:

$$
\begin{aligned}
& I_{u}(t)=\sum_{j \in \Phi_{V B}, j \neq i} P_{V B} h_{u, V B_{j}} r_{u, V B_{j}}^{-\alpha} \\
& =\sum_{j \in \Phi_{V B}, j \neq i} \frac{N P_{B}-P_{C} \lambda_{V B}(t) S_{\text {area }}}{\lambda_{V B}(t) S_{\text {area }}} h_{u, V B_{j}} r_{u, V B_{j}}^{-\alpha}, \forall u .
\end{aligned}
$$


The probability density function (pdf) of the distance $r_{u, \text { min }}$ between the user and its nearest virtual BS satisfies [13]:

$$
f\left(r_{u, \min }\right)=e^{-\lambda_{V B}(t) \pi r_{u, \min }^{2}} \cdot 2 \pi \lambda_{V B}(t) r_{u, \min }
$$

and the pdf of the user's distance $r_{u, V B_{j}}$ from other interfering virtual BSs is:

$$
f\left(r_{u, V B_{j}}\right)=2 r_{u, V B_{j}} / R^{2},
$$

where $R$ is the cell radius of the virtual network. We can find out the average value of interference and SINR received by user $u$ at slot $t$, respectively, given by

$$
\begin{aligned}
& I_{u}^{a v}(t) \\
& =\int_{0}^{\infty} \int_{r_{u, \text { min }}}^{\infty} I_{u}(t) f\left(r_{u, V B_{j}}\right) d r_{u, V B_{j}} f\left(r_{u, \text { min }}\right) d r_{u, \text { min }} \\
& =\int_{0}^{\infty} \frac{2 h_{u, V B_{j}}\left(N P_{B}-P_{C} \lambda_{V B}(t) S_{a r e a}\right)}{(\alpha-2) R^{2}} e^{-\lambda_{V B}(t) \pi r_{u, \text { min }}^{2}} \\
& \cdot 2 \pi \lambda_{V B}(t) r_{u, \text { min }}^{3-\alpha} d r_{u, \text { min }},
\end{aligned}
$$

and

$$
\begin{aligned}
& S I N R_{u}^{a v}(t)=\int_{0}^{\infty} S I N R_{u}(t) f\left(r_{u, \text { min }}\right) d r_{u, \text { min }} \\
& =\int_{0}^{\infty} \frac{\left(N P_{B}-P_{C} \lambda_{V B}(t) S_{a r e a}\right) h_{u, V B_{i}} r_{u, \min }^{-\alpha}}{\lambda_{V B}(t) S_{\text {area }}\left(I_{u}^{a v}(t)+\sigma^{2}\right)} \\
& e^{-\lambda_{V B}(t) \pi r_{u, \min }^{2}} \cdot 2 \pi \lambda_{V B}(t) r_{u, \min } d r_{u, \min }, \forall u \text {. }
\end{aligned}
$$

According to the Shannon formula, the average data rate $R_{u}(t)$ that the RAN slice can provide for user $u$ at slot $t$ is given as:

$$
\begin{aligned}
& R_{u}(t)=E\left[\log _{2}\left(1+S I N R_{u}^{a v}(t)\right)\right] \\
& =\log _{2}\left\{1+\int_{0}^{\infty} \frac{\left(N P_{B}-P_{C} \lambda_{V B}(t) S_{a r e a}\right) h_{u, V B_{i}} r_{u, \text { min }}^{-\alpha}}{\lambda_{V B}(t) S_{\text {area }}\left(I_{u}^{a v}(t)+\sigma^{2}\right)}\right. \\
& \left.f\left(r_{u, \text { min }}\right) d r_{u, \text { min }}\right\} .
\end{aligned}
$$

Consequently, the downlink sum rate of all RAN slices in the virtual network is as follows:

$$
R_{\text {sum }}\left(\lambda_{V B}(t), \lambda_{U}(\mathrm{t})\right)=\lambda_{U}(\mathrm{t}) S_{\text {area }}\left[R_{u}\left(\lambda_{V B}(t)\right)\right] .
$$

At the same time, we can derive the time-average expectation of the rate:

$$
\begin{aligned}
& \bar{R}_{\text {sum }}\left(\lambda_{V B}(t), \lambda_{U}(t)\right) \\
& =\lim _{t \rightarrow \infty} \frac{1}{t} \sum_{\tau=0}^{t-1} E\left\{R_{\text {sum }}\left(\lambda_{V B}(\tau), \lambda_{U}(\tau)\right)\right\} .
\end{aligned}
$$

\section{Delay Analysis of RAN slices}

To analyze the delay of RAN slices, based on Fig. 2, we model the queuing process of data requested by user $u$ as

$$
Q_{u}(t+1)=\max \left[Q_{u}(t)-R_{u}(t), 0\right]+\mathrm{A}_{u}(\mathrm{t}), \forall u \text {. }
$$

Therefore, the average data queue length of all RAN slices in the network can be expressed as follows:

$$
\bar{Q}=\lim _{t \rightarrow \infty} \frac{1}{t} \sum_{\tau=0}^{t-1}\left[\sum_{k=1}^{K(\tau)} E\left\{Q_{k}(\tau)\right\}\right]
$$

where $k$ denotes the $k$ th RAN slice and $K(t)=\lambda_{U}(t) S_{\text {area }}$ denotes the total number of RAN slices in the network.

Using the Little Theorem [14], we can get the average delay of the network as

$$
\bar{D}=\bar{Q} / \gamma
$$

Without loss of generality, we use the length of the data queue to express the delay of the current network. Unless otherwise specified, the delay mentioned in this paper refers to the length of the data queue in the network.

In this paper, we study the problem of rate-delay tradeoff given the network is stable. A network is stable when all these discrete queues $Q_{k}(t)$ are mean rate stable, that is, satisfying the following condition [15]:

$$
\lim _{t \rightarrow \infty} \frac{E\left\{\left|Q_{k}(t)\right|\right\}}{t}=0 .
$$

\section{Problem Formulation AND Solution}

According to (1), the power in the network is constant, so when the sum rate increases, the overall energy efficiency of the network increases. Therefore, in this section, we study the sum rate maximization of the RAN slices.

We develop a delay-constrained sum rate maximization problem. Mathematically, the problem can be formulated as

$$
\begin{array}{ll}
\max & \bar{R}_{\text {sum }}\left(\lambda_{V B}(t), \lambda_{U}(t)\right) \\
\text { s.t. } & C 1: \bar{P}_{V B} \leq P_{V B}^{a v}, \forall k, \\
& C 2: Q_{k}(t) \leq \beta, \forall k, t, \\
& C 3: \lambda_{V B}(t) \geq 0, \forall t, \\
& C 4: P_{V B}(t) \geq 0, \forall t,
\end{array}
$$

where $P_{V B}^{a v}$ and $\beta$ denote the threshold values of the average power consumption by virtual BSs and the maximum tolerable delay requested by users, respectively. $P_{V B}^{a v}$ is used to ensure that the physical BSs have sufficient energy to maintain and operate normally; and $\beta$ is used to guarantee the users' QoS. In addition, $C 1$ constraints the average power consumption by virtual BSs. According to (15), $C 2$ guarantees the stability of queues and limits the equivalent queue length in RAN slices. $C 3$ and $C 4$ give the non-negativity of the virtual BS density and the power.

Based on the general Lyapunov theory [15], we transform the average power constraint $C 1$ in (18) into a virtual power queue stable problem. The virtual power queue is defined as $G_{k}(t)$, where $G_{k}(0)=0$ and

$$
G_{k}(t+1)=\max \left[G_{k}(t)+P_{V B}(\mathrm{t})-P_{V B}^{a v}, 0\right], \forall k, t .
$$

Combining (14) and (15), we can rewritten the $C 2$ in (18) equivalently as the following constraint $\tilde{C} 2$ :

$Q_{k}(t+1)=\left\{\begin{array}{c}\max \left[Q_{k}(t)-R_{k}(t), 0\right], \text { if } Q_{k}(t)>\beta \\ \max \left[Q_{k}(t)-R_{k}(t), 0\right]+A_{k}(t), \text { e.w. }\end{array}\right.$ 
Considering (3), $C 4$ can be equivalently transformed into the following constraint $\tilde{C} 4$ :

$$
\lambda_{V B}(\mathrm{t}) \leq \frac{N P_{B}}{P_{C} S_{\text {area }}} .
$$

Then, (18) can be further recast equivalently to

$$
\begin{array}{ll}
\max & \bar{R}_{\text {sum }}\left(\lambda_{V B}(t), \lambda_{U}(t)\right) \\
\text { s.t. } & \tilde{C} 2, C 3, \tilde{C} 4, \\
& C 1: G_{k}(t) \text { is mean rate stable }, \forall k .
\end{array}
$$

To tackle $C 1$ and $\tilde{C} 2$, a combined vector, $\Theta(t)=$ $[Q(t), G(t)]$, is defined to represent queuing states of all queues, where $Q(t)$ and $G(t)$ are virtual queues sets.

Then, we can get the conditional Lyapunov drift $\triangle[\Theta(t)]$ :

$$
\triangle[\Theta(t)]=E\{L[\Theta(t+1)]-L[\Theta(t)] \mid \Theta(t)\},
$$

where the $L[\Theta(t)]$ is Lyapunov function given by

$$
L[\Theta(t)]=\frac{1}{2} \sum_{k=1}^{K(t)} Q_{k}(t)^{2}+\frac{1}{2} \sum_{k=1}^{K(t)} G_{k}(t)^{2} .
$$

The drift-plus-penalty expression here can be obtained as

$$
F(t)=\Delta[\Theta(t)]-V E\left[R_{\text {sum }}(t)\right],
$$

where $V \geq 0$ is the weight factor between the rate and delay.

We have the following inequality about $F(t)$ by using Lyapunov optimization theory [15],

$$
\begin{aligned}
& F(t) \leq B+\sum_{k=1}^{K(t)} \xi\left[Q_{k}(t)-\beta\right] Q_{k}(t) E\left[A_{k}(t) \mid \Theta(t)\right] \\
& +\sum_{k=1}^{K(t)} G_{k}(t) E\left[P_{V B}(\mathrm{t})-P_{V B}^{a v} \mid \Theta(t)\right] \\
& -\sum_{k=1}^{K(t)} Q_{k}(t) E\left[R_{k}(t) \mid \Theta(t)\right]-V E\left[R_{\text {sum }}(t) \mid \Theta(t)\right],
\end{aligned}
$$

where $B>0$ is a limited constant and satisfies the following condition:

$$
\begin{aligned}
& B \geq \frac{1}{2} \sum_{k=1}^{K(t)} E\left\{\left[\xi\left[Q_{k}(t)-\beta\right] A_{k}(t)\right]^{2}+R_{k}(t)^{2} \mid \Theta(t)\right\} \\
& +\frac{1}{2} \sum_{k=1}^{K(t)}\left\{\left[P_{V B}(\mathrm{t})-P_{V B}^{a v}\right]^{2} \mid \Theta(t)\right\},
\end{aligned}
$$

where $\xi$ is a function of $Q_{k}(t)-\beta$ and satisfies: $Q_{k}(t)-\beta \leq 0$, $\xi\left[Q_{k}(t)-\beta\right]=1$; otherwise, $\xi\left[Q_{k}(t)-\beta\right]=0$, which satisfies $\tilde{C} 2$ in (22).

Specifically, with the help of the stochastic optimization theory [15], the optimal solution of the above problem (22) can be obtained by minimizing the upper bound of $F(t)$ slot by slot, i.e., solving the problem (28) below:

$$
\begin{aligned}
& \min \sum_{k=1}^{K(t)}\left\{G_{k}(t)\left[P_{V B}(\mathrm{t})-P_{V B}^{a v}\right]-Q_{k}(t) R_{k}(t)\right. \\
& \left.+\xi\left[Q_{k}(t)-\beta\right] Q_{k}(t) A_{k}(t)\right\}-V\left[R_{\text {sum }}(t)\right] \\
& \text { s.t. } C 3: \lambda_{V B}(t) \geq 0, \forall t, \\
& \quad \tilde{C} 4: \lambda_{V B}(\mathrm{t}) \leq \frac{N P_{B}}{P_{C} S_{\text {area }}}, \forall t .
\end{aligned}
$$

At this point, we have transformed the original difficult problem (18) into the problem (28) that is easy to solve.

Therefore, we can propose an effective virtual resource allocation algorithm based on the classic drift-plus-penalty algorithm, summarized in Algorithm 1. In particular, the solution to equation (28) can be found using a simple onedimensional search.

\footnotetext{
Algorithm 1 Dynamic virtual resource allocation algorithm to solve (28).

1: Initialization: $Q_{k}(0)=0, G_{k}(0)=0$, and $R_{\text {sum }}(0)=0, \forall k$.

2: Repeat:

3: Update virtual resource allocation $\lambda_{V B}(t)$ according to (28).

4: Let $t=t+1$.

5: Update $Q_{k}(t), G_{k}(t)$ and $R_{\text {sum }}(t)$ according to (20), (19) and (12), respectively.

6: Stop when $t=T$, where $T$ is the total number of time slots.
}

\section{TRADEOFF BETWEEN RATE AND DELAY}

In this section, the upper bounds of the sum rate and delay, as well as their tradeoff will be derived.

Theorem 1: The sum rate in (18) is bounded by

$$
R_{\text {sum }}^{\text {opt }} \geq \bar{R}_{\text {sum }}\left(\lambda_{V B}(t), \lambda_{U}(\mathrm{t})\right) \geq R_{\text {sum }}^{\text {opt }}-\frac{B}{V} .
$$

Proof: Assume that $R_{k}^{*}(t), P_{V B}^{*}(t)$ and $R_{\text {sum }}^{*}(t)$ result from an feasible virtual BS allocation policy $\lambda_{V B}(t)$ of problem (18) in slot $t$. The following conditions for any $\delta>0$ and $\varepsilon>0$ can be obtained according to the stochastic optimization theory [15]:

$$
\begin{gathered}
E\left[A_{k}(t)-R_{k}^{*}(t) \mid \Theta(t)\right]=E\left[A_{k}(t)-R_{k}^{*}(t)\right] \leq \delta \\
E\left[R_{\text {sum }}^{*}(t) \mid \Theta(t)\right]=E\left[R_{\text {sum }}^{*}(t)\right] \geq R_{\text {sum }}^{\text {opt }}+\delta \\
E\left[R_{k}^{*}(t) \mid \Theta(t)\right]=E\left[R_{k}^{*}(t)\right] \geq \gamma_{k}+\varepsilon \\
E\left[P_{V B}(\mathrm{t})-P_{V B}^{a v}(t) \mid \Theta(t)\right]=E\left[P_{V B}(\mathrm{t})-P_{V B}^{a v}(t)\right] \leq \delta
\end{gathered}
$$

where $\gamma_{k}$ is the data arrival rate of each RAN slice.

Substituting (30)-(33) into the right-hand side of (26) and letting $\delta \rightarrow 0$, we have the following inequality:

$$
\begin{aligned}
& \Delta[\Theta(t)]-V E\left[R_{\text {sum }}(t) \mid \Theta_{t}\right] \\
& \leq B+\sum_{k=1}^{K(t)}\left\{\xi\left[Q_{k}(t)-\beta\right]-1\right\} Q_{k}(t) \gamma_{k} \\
& -\varepsilon \sum_{k=1}^{K(t)} Q_{k}(t)-V R_{\text {sum }}^{\text {opt }}(t) .
\end{aligned}
$$


Taking expectation for both sides of (34), and then using telescope sum over $t \in\{0,1, \ldots, H-1\}$ for the result as well as taking the fact that $Q_{k}(t) \geq 0$ and $\xi\left[Q_{k}(t)-\beta\right]-1 \leq 0$ into consideration, we have

$$
\begin{aligned}
& E\{L[\Theta(H)]\}-E\{L[\Theta(0)]\}-V \sum_{t=0}^{H-1} E\left[R_{\text {sum }}(t)\right] \\
& \leq H B-H V R_{\text {sum }}^{\text {opt }},
\end{aligned}
$$

where $H$ represents the number of the time slots.

Dividing both sides of the above inequality by $\mathrm{VH}$ yields the following:

$$
\frac{-E\{L[\Theta(0)]\}}{V H}-\frac{1}{H} \sum_{t=0}^{H-1} E\left[R_{\text {sum }}(t)\right] \leq \frac{B}{V}-R_{\text {sum }}^{\text {opt }} .
$$

Let $H \rightarrow \infty$ and consider equation $E\{L[\Theta(0)]\} \geq 0$, we can obtain the following conclusion

$$
\begin{aligned}
& \bar{R}_{\text {sum }}\left(\lambda_{V B}(t), \lambda_{U}(\mathrm{t})\right)=\frac{1}{H} \sum_{t=0}^{H-1} E\left[R_{\text {sum }}(t)\right] \\
& \geq R_{\text {sum }}^{\text {opt }}-\frac{B}{V} .
\end{aligned}
$$

Thus, the (29) can be proved.

Theorem 2: The average queue length is bounded by

$$
\begin{aligned}
& \bar{Q}=\lim _{H \rightarrow \infty} \frac{1}{H} \sum_{t=0}^{H-1}\left[\sum_{k=1}^{K(t)} E\left\{Q_{k}(t)\right\}\right] \\
& \leq \min \left\{\lambda_{u} S_{\text {area }} \beta, \frac{B+V\left(\lambda_{u} S_{\text {area }} R_{k}^{\max }-R_{\text {sum }}^{\text {opt }}\right)}{\varepsilon}\right\},
\end{aligned}
$$

where $R_{k}^{\max }$ is the rate threshold available for a RAN slice, and $\lambda_{u}$ is the time-average expectation of $\lambda_{U}(t)$.

Proof: When $Q_{k}(t) \leq \beta$, take expectation for (34) while using telescoping sum over $t \in\{0,1, \ldots, H-1\}$ for the result. Considering $\xi\left[Q_{k}(t)-\beta\right]=1$, we have

$$
\begin{aligned}
& E\{L[\Theta(H)]\}-E\{L[\Theta(0)]\}-V \sum_{t=0}^{H-1} E\left[R_{\text {sum }}(t)\right] \\
& \leq H B-H V R_{\text {sum }}^{\text {opt }}-\varepsilon \sum_{t=0}^{H-1} \sum_{k=1}^{K(t)} E\left[Q_{k}(t)\right] .
\end{aligned}
$$

Dividing both sides of the above inequality by $\varepsilon H$ and taking $H \rightarrow \infty$ we have:

$$
\begin{aligned}
& \bar{Q}=\lim _{H \rightarrow \infty} \frac{1}{H} \sum_{t=0}^{H-1}\left\{\sum_{k=1}^{K(t)} E\left[Q_{k}(t)\right]\right\} \\
& \leq \frac{B-V R_{\text {sum }}^{\text {opt }}}{\varepsilon}+\frac{V}{\varepsilon} \lim _{H \rightarrow \infty} \frac{1}{H} \sum_{t=0}^{H-1} E\left[R_{\text {sum }}(t)\right],
\end{aligned}
$$

where

$$
\lim _{H \rightarrow \infty} \frac{1}{H} \sum_{t=0}^{H-1} E\left[R_{\text {sum }}(t)\right] \leq R_{\text {sum }}^{\max } .
$$

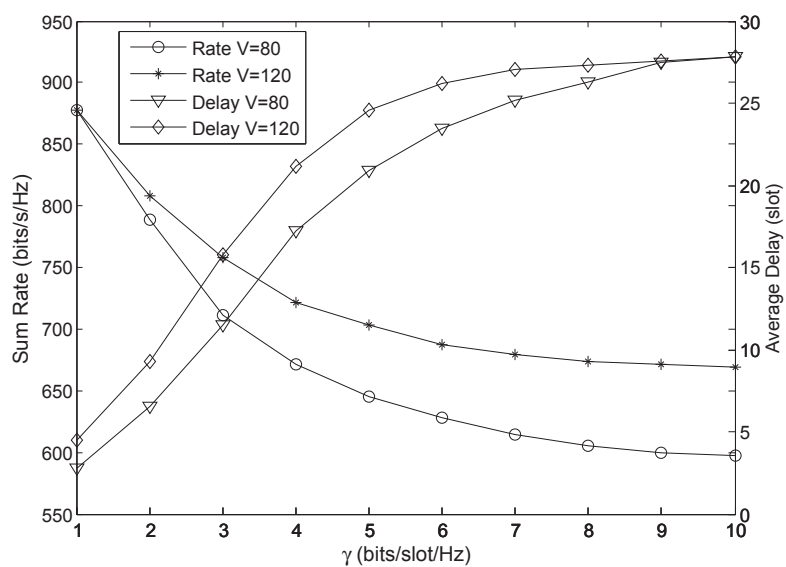

Figure 3. Sum rate and average delay versus $\gamma$.

Considering (12), we have:

$$
\begin{aligned}
& \bar{Q}=\lim _{H \rightarrow \infty} \frac{1}{H} \sum_{t=0}^{H-1}\left\{\sum_{k=1}^{K(t)} E\left[Q_{k}(t)\right]\right\} \\
& \leq \frac{B+V\left(\lambda_{u} S_{\text {area }} R_{k}^{\max }-R_{\text {sum }}^{\text {opt }}\right)}{\varepsilon} .
\end{aligned}
$$

Furthermore, we can easily obtain the following conclusion when $Q_{k}(t) \geq \beta$, or

$$
\bar{Q}=\lambda_{u} S_{\text {area }} \beta .
$$

Combining both (42)and (43) completes the proof.

\section{NUMERICAL RESULTS AND DISCUSSIONS}

In this section, extensive numerical evaluation results are provided to validate the theoretical findings. The transmit power of a $\mathrm{BS}$ is assumed to be $P_{B}=20 \mathrm{~W}$ and the number of physical BSs is $N=2$. In addition, we set $\alpha=4$, $\lambda_{u}=0.000125\left(/ \mathrm{m}^{2}\right)$ and $S_{\text {area }}=2 \mathrm{~km} \times 2 \mathrm{~km}$. Moreover, we use 10,000 slots to approximate $t \rightarrow \infty$.

Fig. 3 indicates the network performance versus data arrival rate $\gamma$ with different settings, where $V$ is the control factor of the tradeoff between the sum rate and delay. The average delay of one RAN slice increases exponentially with $\gamma$ at first and then tends to be stable when the network queueing length exceeds the limit of $\beta$. Similarly, the sum rate decreases with $\gamma$ and eventually converges because of the limit of $P_{V B}^{a v}$ and $\beta$.

Fig. 4 illustrates the impact of $V$ on the sum rate and delay for RAN slices under different $\gamma$. The sum rate tends to converge with the increase of $V$. When $V$ approaches infinity, the sum rate converges to the optimal rate of the system. Then the average delay of one RAN slice shows a linear growth trend with the increase of $V$ because the queue length does not exceed the limit of $\beta$. In addition, we can see that $\gamma$ has a negative impact on the network performance, that is, when $V$ is the same, the larger $\gamma$, the lower the sum rate as well 


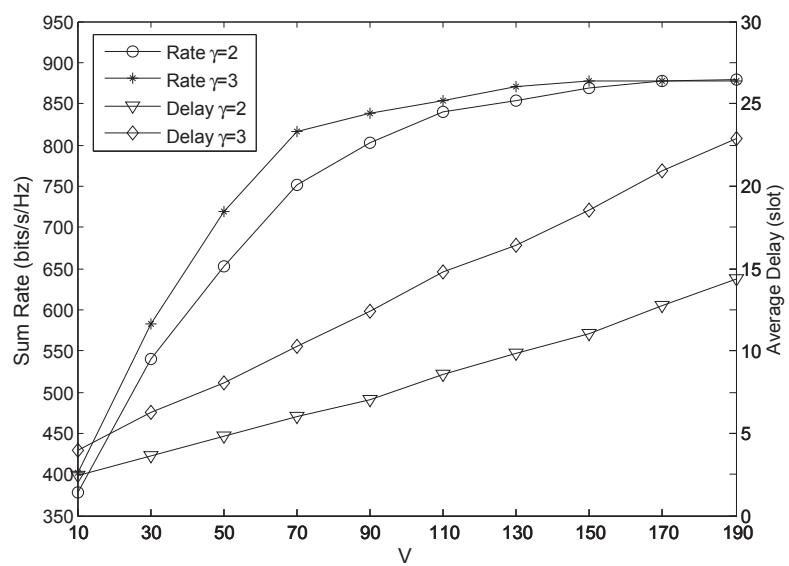

Figure 4. Sum rate and average delay versus $V$.

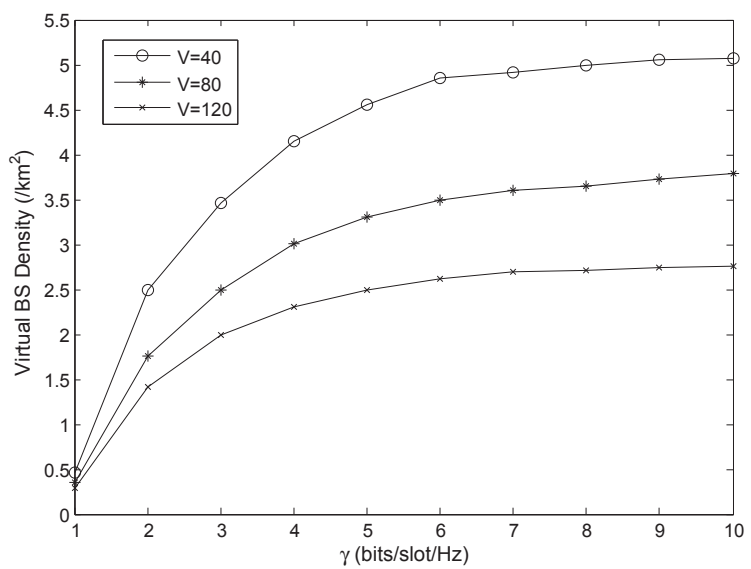

Figure 5. Virtual BS density versus data arrival rate.

as the larger the average delay. Finally, as we can see, the optimization of the sum rate can be achieved at the cost of the delay. The lower the delay, the lower the sum rate. There is a clear performance trade-off between the two metrics, as discussed in the previous section.

Fig. 5 describes virtual BS density versus data arrival rate with different settings of $V$. We can see that the optimal virtual BS density increases at the beginning, and then tend to be stable because of the limit of $P_{V B}^{a v}$ and $\beta$ with $\gamma$. That is, when the data arrival rate in the network increases, we can increase system performance by increasing the number of virtual BS properly in the system. In addition, $V$ has a positive impact on network performance. The network can transmit larger amount of data for users in the same virtual BS deployment with increased of $V$.

\section{CONCLUSiOnS AND Future Work}

In this paper, the rate-delay tradeoff problem is investigated in RAN slices with randomly distributed BSs and UEs. The problem is formulated as a stochastic optimization problem to optimize the sum data rate with the constraint on the delay. Afterwards, it is solved using the Lyapunov optimization. Finally, the theoretical bounds for both the sum rate and delay are derived, which reveal the way of adjusting the parameter $V$ to get a performance tradeoff. Future work is in progress to consider caching and computing resource allocation in RAN slices.

\section{ACKNOWLEDGMENT}

This work was supported in part by National Natural Science Foundation of China (61771358), National Natural Science Foundation of Shaanxi Province (2018JM6052), Intergovernmental International Cooperation on Science and Technology Innovation (2016YFE0122900), and the 111 Project (B08038).

\section{REFERENCES}

[1] Y. Zhang, C. Jiang, L. Song, W. Saad, Z. Dawy and Z. Han, "Complementary Investment of Infrastructure and Service Providers in Wireless Network Virtualization," in Proc.GLOBECOM, Washington DC,U.S., Dec. 2016, pp. 1-6.

[2] C. Liang and F. R. Yu, "Wireless virtualization for next generation mobile cellular networks, "IEEE Wireless Communications., vol. 22, no. 1, pp. 61-69, Feb. 2015.

[3] M. Richart, J. Baliosian, J. Serrat and J. L. Gorricho, "Resource Slicing in Virtual Wireless Networks: A Survey," in IEEE Transactions on Network and Service Management, vol. 13, no. 3, pp. 462-476, Sept. 2016.

[4] A. Ksentini and N. Nikaein, "Toward Enforcing Network Slicing on RAN: Flexibility and Resources Abstraction," in IEEE Communications Magazine, vol. 55, no. 6, pp. 102-108, 2017.

[5] O. Sallent, J. Perez-Romero, R. Ferrus and R. Agusti, "On Radio Access Network Slicing from a Radio Resource Management Perspective,' IEEE Wireless Commun., vol. 24, no. 5, pp. 166-174, October 2017.

[6] P. Caballero, A. Banchs, G. de Veciana and X. Costa-Prez, "MultiTenant Radio Access Network Slicing: Statistical Multiplexing of Spatial Loads," in IEEE/ACM Transactions on Networking, vol. 25, no. 5, pp. 3044-3058, Oct. 2017.

[7] S. Tao, L. Gu, D. Zeng, H. Jin and K. Hu, "Fairness-aware dynamic rate control and flow scheduling for network function virtualization," 2017 IEEE/ACM 25th International Symposium on Quality of Service (IWQoS), Vilanova i la Geltru, 2017, pp. 1-6.

[8] Y. L. Lee, J. Loo, T. C. Chuah and L. C. Wang, "Dynamic Network Slicing for Multitenant Heterogeneous Cloud Radio Access Networks," in IEEE Transactions on Wireless Communications, vol. 17, no. 4, pp. 2146-2161, April 2018.

[9] L. Zhang, J. Liu, and K. Yang, "Quality of Service Modelling of Virtualized Wireless Networks: A Network Calculus Approach," Springer Mobile Networks and Applications, vol. 19, no. 4, pp. 572-582, 2014.

[10] L. Tang, X. Zhang, H. Xiang, Y. Sun and M. Peng, "Joint resource allocation and caching placement for network slicing in fog radio access networks," 2017 IEEE 18th International Workshop on Signal Processing Advances in Wireless Communications (SPAWC), Sapporo, 2017, pp. 1-6.

[11] C. She and C. Yang, "Energy Efficiency and Delay in Wireless Systems: Is Their Relation Always a Tradeoff?,' IEEE Trans. Wireless Commun. vol. 15, no. 11, pp. 7215-7228, Nov. 2016.

[12] Q. Shi, L. Zhao, Y. Zhang, G. Zheng, F. R. Yu and H. H. Chen, ’EnergyEfficiency Versus Delay Tradeoff in Wireless Networks Virtualization," in IEEE Transactions on Vehicular Technology, vol. 67, no. 1, pp. 837841, Jan. 2018.

[13] J. G. Andrews, F. Baccelli and R. K. Ganti, "A Tractable Approach to Coverage and Rate in Cellular Networks," IEEE Trans. Commun., vol. 59, no. 11, pp. 3122-3134, November 2011.

[14] D. P. Bertsekas and R. G. Gallager, Data Networks (2nd edition). Prentice Hall, 1992.

[15] M. J. Neely, Stochastic Network Optimization with Application to Communication and Queueing Systems. San Rafael, CA, USA: Morgan and Claypool, 2010 